\title{
Avaliação da percepção em farmacologia dos cirurgiões-dentistas do município de Patos-PB
}

\author{
Evaluation of perception in pharmacology of the dental surgeries of the \\ municipality of Patos-PB \\ Evaluación de percepción in pharmacología de la dental surgeries de la \\ municipalidad de Patos-PB \\ Moan Jéfter Fernandes COSTA \\ Basílio Rodrigues VIEIRA2 \\ Paulyana Pryscilla de Melo FREIRE ${ }^{3}$ \\ Francys Deize Fernandes COSTA ${ }^{4}$ \\ Maria Angélica Sátyro Gomes ALVES ${ }^{5}$ \\ Gymenna Maria Tenório GUÊNES ${ }^{6}$
}

${ }^{l}$ Cirurgião-Dentista pela Universidade Federal de Campina Grande (UFCG), Mestrando do em Saúde Coletiva pela Universidade Federal do Rio Grande do Norte (UFRN). 59200-000 Santa Cruz-RN, Brasil

${ }^{2}$ Cirurgião-Dentista pela Universidade Federal de Campina Grande (UFCG), Mestrando em Ciências Odontológica pela Universidade Federal da Paraíba (UFPB), 58057-270 João Pessoa-PB, Brasil

${ }^{3}$ Cirurgiã-Dentista pela Universidade Federal de Campina Grande (UFCG), Especialista em Ortodontia pelo NEAO 58064-550 João Pessoa-PB, Brasil

${ }^{4}$ Cirurgiã-Dentista pela Universidade Federal de Campina Grande (UFCG) 58708-110 Patos-PB, Brasil

${ }^{5}$ Farmacêutica, Doutora em Produtos Naturais e Sintéticos Bioativos pela Universidade Federal da Paraíba (UFPB),

Professora Adjunto de Fisiologia e Bioquímica Geral. da Universidade Federal de Campina Grande (UFCG) 58708-110 Patos-PB, Brasil

${ }^{6}$ Cirurgiã-Dentista, Doutora em Odontologia pela Faculdade de Odontologia do Pernambuco (FOP-UPE)

Professora Adjunto de Dentística e Clinica Multidisciplinar da Universidade Federal de Campina Grande (UFCG), 58708-110 Patos-PB, Brasil

\section{Resumo}

Devido ao fato dos cirurgiões-dentistas requererem de um vasto arsenal de fármacos que são imprescindíveis para muitos procedimentos durante a clínica odontológica, faz-se necessário que possuam um excelente nível de informação sobre a farmacologia destes medicamentos. O objetivo é uma análise crítica do conhecimento dos cirurgiões-dentistas em relação ao uso e aplicação correta de diversos fármacos na clínica odontológica. Através de um estudo transversal de abordagem indutiva com 100 cirurgiõesdentistas da cidade de Patos-Paraíba, foram analisados aspectos relacionados a prescrição de antimicrobianos, anti-inflamatórios, anestésicos e outros, vantagens quanto ao efeito e contraindicações, analise da conduta terapêutica e autoconhecimento. Para análise dos dados utilizou-se técnicas de estatística descritiva e inferencial. Concluiu-se que em geral os cirurgiões-dentistas entrevistados apresentam um déficit em relação a prescrição correta de determinados fármacos, mostrando ainda que esses mesmos profissionais admitem ter um conhecimento limitado na área farmacológica, evidenciando a necessidade do aprimoramento e atualização do conhecimento sobre o uso correto e efetivo de uma grande parcela de medicamentos de uso rotineiro.

Descritores: Odontologia; Prescrições de Medicamentos; Farmacologia.

\begin{abstract}
Currently it appears that the dentist uses a large arsenal of drugs, essential for many dental procedures, so, like doctors, the dental surgeon must have an excellent level of information about the pharmacology of many drugs. The objective is to critically analyze the knowledge of dental professionals regarding the correct application of drugs. A cross-sectional study with inductive approach and comparative procedures was carried out, where 100 dental surgeons answered a questionnaire in which the following aspects were analyzed: prescription of antimicrobials, anti-inflammatories, anesthetics and others, regarding the advantages in terms of effect and contraindications, analysis of therapeutic behavior and self-knowledge. Descriptive and inferential statistical techniques were used to analyze the data. Through this research, it was possible to verify that the dental surgeons interviewed present a deficit in relation to the correct prescription of certain drugs. The data still showed that the majority of professionals interviewed consider their knowledge in the pharmacological area insufficient. It could be verified that the limitation of the participants evidences the need to improve the professionals' knowledge about the rational use of these drugs.
\end{abstract}

Descriptors: Dentistry; Drug Prescriptions; Pharmacology.

\section{Resumen}

Debido al hecho de que los cirujanos-dentistas requieran de un vasto arsenal de fármacos que son imprescindibles para muchos procedimientos durante la clínica odontológica, se hace necesario que tengan un excelente nivel de información sobre la farmacología de estos medicamentos. El objetivo es un análisis crítico del conocimiento de los cirujanos-dentistas en relación al uso y aplicación correcta de diversos fármacos en la clínica odontológica. A través de un estudio transversal de abordaje inductivo con 100 cirujanos-dentistas de la ciudad de Patos-Paraíba, se analizaron aspectos relacionados con la prescripción de antimicrobianos, antiinflamatorios, anestésicos y otros, ventajas en cuanto al efecto y contraindicaciones, análisis de la conducta terapéutica y autoconocimiento. Para el análisis de los datos se utilizaron técnicas de estadística descriptiva e inferencial. Se concluyó que en general los cirujanos-dentistas entrevistados presentan un déficit en relación a la prescripción correcta de determinados fármacos, mostrando además que esos mismos profesionales admite tener un conocimiento limitado en el área farmacológica, evidenciando la necesidad del perfeccionamiento y actualización del conocimiento sobre el uso es correcto y efectivo de una gran parte de medicamentos de uso rutinario.

Descriptores: Odontología; Prescripciones de Medicamentos; Farmacología.

\section{INTRODUÇÃO}

Os medicamentos, em uma forma geral, são objetos de pesquisa em todo mundo, desde todos os mecanismos associados à sua produção até ao consumo. Porém, grande maioria dessas pesquisas centra-se na prática médica, todavia, atualmente o cirurgião dentista faz uso de um vasto arsenal de fármacos específicos, que vão de administração oral a endovenosa, em decorrência da abrangência de procedimentos realizados no consultório odontológico ou mesmo em ambiente hospitalar ${ }^{1,2}$.

Assim percebe-se que a farmacologia dos medicamentos e seus distintos mecanismos de ação são de extrema importância para o tratamento odontológico como meio paliativo de diferentes afecções e, sobretudo como instrumento auxiliar nos procedimentos realizados na 
cavidade bucal ${ }^{3,4}$.

É preciso que o cirurgião-dentista entenda os problemas e preocupações associados à prática irracional do uso e administração de medicamentos, para que racionalize o seu uso e indicação durante a prática odontológica, conhecendo o papel essencial de cada medicamento no processo de prevenção e tratamento, para que alie de maneira satisfatória a seleção e indicação aos mecanismos de interações medicamentosas, efeitos nocivos e controle dos fármacos 5 .

São escassas pesquisas que buscam identificar os níveis de informações sobre farmacologia e o embasamento dos cirurgiões-dentistas a respeito dos medicamentos da prática odontológica. Com base no exposto, o propósito deste trabalho foi avaliar o nível de informação do ponto de vista técnico e científico dos cirurgiões-dentistas (CD) do município de Patos-PB sobre farmacologia.

\section{MATERIAL E MÉTODO}

O presente estudo foi desenvolvido utilizando como população os cirurgiões-dentistas da cidade de Patos-PB. Após submissão e aprovação do Comitê de Ética em Pesquisa do Centro de Saúde e Tecnologia Rural da Universidade Federal de Campina Grande (UFCG/CSTR) sob o protocolo CEP n ${ }^{\circ}$ 19-2011 participantes do estudo foram entrevistados, garantindo-lhes o sigilo da informação e desautorizando qualquer forma de coação ou pressão pela participação voluntária por parte desse indivíduo. Portanto trata-se de uma pesquisa do tipo descritiva, exploratória e transversal, com procedimentos comparativos e estatísticos, tendo como instrumento para avaliação dos entrevistados, um questionário, realizado de forma indutiva.

O cálculo amostral levou em consideração um erro tipo I de $5 \%$ bicaudal $(\alpha=0,05 \%, z=1,96)$ com uma proporção de $50 \%$, tendo em vista que nenhum outro estudo foi realizado nas mesmas situações, sendo uma proporção desconhecida. A população da qual foi extraído a amostra levou em consideração o número de cirurgiões-dentistas com cadastro no Conselho Regional de Odontologia da Paraíba (CRO-PB) atuando no setor público e privado da cidade de Patos-PB $(\mathrm{N}=142)$. Com base no exposto chegou-se a amostra de mínima de 94 cirurgiões-dentistas para que a amostra seja comparativa e representativa ao número total da população.

Foi realizado um estudo piloto com 20 questionários com o intuito de testar o entendimento dos métodos e instrumentos. Os cirurgiões-dentistas que participaram do estudo piloto não fizeram parte da amostra final do estudo. Selecionou-se por meio de amostragem aleatória simples 100 CD's que exerciam atividade profissional no setor privado e/ou público que apresentassem participação voluntária.

Os dados obtidos foram tabulados, agrupados e analisados por meio de estatística descritiva mediante a utilização do software Statistical Package for Social Science (SPSS) v.2.0.

\section{RESULTADOS}

A amostra do presente estudo foi composta por 100 cirurgiões-dentistas. Com relação à autopercepção sobre a farmacologia na vida profissional, a maioria dos cirurgiõesdentistas alegou ser muito importante, perfazendo um total de $97 \%$, os $3 \%$ restante responderam ser razoavelmente importante. Com relação a participação em cursos de reciclagem voltadas para a área de farmacologia, 63\% afirmaram que já participaram, tendo em vista que $62 \%$ afirmaram que o ensino de farmacologia na graduação foi insuficiente. Quando perguntados sobre a autoavaliação dos conhecimentos em farmacologia, a maioria dos profissionais (53\%) disse ser regular, seguido por suficiente. A descrição completa da autopercepção dos cirurgiões-dentistas está apresentada na Tabela 1 .
Tabela 1. Autopercepção dos cirurgiões-dentistas sobre os conhecimentos de Farmacologia

\begin{tabular}{lc}
\hline \multicolumn{3}{l}{ A Farmacologia se faz importante na prática odontológica? } \\
\hline Muito importante & $97 \%$ \\
Importante & $0 \%$ \\
Razoavelmente importante & $3 \%$ \\
Não importante & $0 \%$ \\
\hline Participação em cursos de & reciclagem voltados para \\
Farmacologia & \\
\hline Sim & $63 \%$ \\
Não & $37 \%$ \\
\hline O ensino de Farmacologia na graduação foi suficiente? \\
\hline Sim & $37 \%$ \\
Não & $62 \%$ \\
Não responderam & $1 \%$ \\
\hline Domínio dos conhecimentos de Farmacologia \\
\hline Suficiente & $43 \%$ \\
Regular & $53 \%$ \\
Insuficiente & $2 \%$ \\
Não responderam & $2 \%$ \\
\hline
\end{tabular}

Quando avaliados sobre o uso os antimicrobianos mais prescritos em primeira escolha, a maior parcela dos entrevistados afirmou prescrever Amoxicilinas (93\%) ou Ampicilinas (7\%). Em caso de segunda escolha, a exemplo de pacientes que são alérgicos aos derivados de penicilinas, $44 \%$ dos profissionais afirmaram prescrever Clindamicina. Os outros medicamentos citados como escolhas estão relatados na Tabela 2. Ainda com relação ao uso de antibióticos, com relação ao uso com finalidade profilática $91 \%$ dos entrevistados afirmaram indicar a profilaxia antibiótica para prevenir a endocardite bacteriana, 9\% dos participantes responderam que não indicariam a profilaxia antibiótica para nenhum tipo de paciente.

Tabela 2. Seleção e uso de antimicrobianos

\begin{tabular}{lc}
\hline \multicolumn{2}{l}{ Antibióticos de primeira escolha } \\
\hline Amoxicilina & $93 \%$ \\
Ampicilina & $7 \%$ \\
Outros & $0 \%$ \\
\hline Antibióticos para alérgicos à penicilina \\
\hline Amoxicilina & $3 \%$ \\
Ampicilina & $1 \%$ \\
Clindamicina & $44 \%$ \\
Eritromicina & $41 \%$ \\
Outros & $6 \%$ \\
Não sabem & $3 \%$ \\
\hline
\end{tabular}

Com relação aos antiinflamatórios preferido para uso, os fármacos mais citados foram a nimesulida (55\%), diclofenacos (26\%), ibuprofeno (15\%), meloxicam $(2 \%)$ e $2 \%$ outros tipos de medicamentos (Tabela 3).

No que diz respeito à prescrição de medicamentos, a expressiva maioria dos profissionais $(97 \%)$ relatam realizar por escrito, e 3\% realizam de forma oral. Quando perguntando sobre os critérios de prescrição de um fármaco 46\% relataram escolherem mediante disponibilidade na UBS, porém motivos como nível socioeconômico do paciente (30\%), relação nacional de medicamentos essenciais (RENAME) (16\%) e pelo valor econômico (4\%) também foram elencados pelos profissionais. Quando questionados sobre a credibilidade as propagandas farmacêuticas veiculadas na mídia $74 \%$ dos entrevistados conferem moderada credibilidade, $2 \%$ alta, $18 \%$ baixa, $6 \%$ foram indiferentes (Tabela 4).

Em referência aos sais anestésicos de uso odontológico algumas questões foram expressivas, a quantidade de solução anestésica contendo adrenalina aplicada em cardiopatas numa única sessão, a maioria dos profissionais respondeu que aplica no máximo 2 tubetes. Perguntou-se ainda sobre a solução 
anestésica não indicada para gestantes, onde $48 \%$ respondeu prilocaína. Os fatores citados com relação a sais anestésicos estão presentes na Tabela 5 .

Tabela 3. Seleção e uso de anti-inflamatórios

\begin{tabular}{lc}
\hline Anti-inflamatórios preferidos para prescrição \\
\hline Nimesulida & $55 \%$ \\
Diclofenaco & $26 \%$ \\
Ibuprofeno & $15 \%$ \\
Meloxicam & $2 \%$ \\
Outros & $2 \%$ \\
\hline
\end{tabular}

Tabela 4. Forma e critérios para prescrição de um fármaco

\begin{tabular}{lc}
\hline \multicolumn{2}{l}{ Forma de prescrição do fármaco } \\
\hline Escrito & $97 \%$ \\
Oral & $3 \%$ \\
\hline Critério de prescrição do fármaco \\
\hline Disponível na UBS & $46 \%$ \\
Renda do paciente & $30 \%$ \\
RENAME & $16 \%$ \\
Preço do fármaco & $4 \%$ \\
\hline Credibilidade das propagandas farmacêuticas \\
\hline Alta & $2 \%$ \\
Moderada & $74 \%$ \\
Baixa & $18 \%$ \\
Indiferente & $6 \%$ \\
\hline
\end{tabular}

Tabela 5. Seleção e uso de anestésicos locais

\begin{tabular}{lcl}
\hline $\begin{array}{l}\text { Quantidades de tubetes com } \\
\text { cardiopatas por sessão }\end{array}$ & adrenalina em pacientes \\
\hline 1 tubete & $26 \%$ \\
2 tubetes & $50 \%$ \\
3 tubetes & $12 \%$ \\
4 tubetes & $5 \%$ \\
5 tubetes & $2 \%$ \\
Não sabiam & $5 \%$ \\
\hline Solução anestésica não indicada para gestantes \\
\hline Articaína & $13 \%$ \\
Bupivacaína & $9 \%$ \\
Lidocaína & $8 \%$ \\
Mepivacaína & $8 \%$ \\
Prilocaína & $48 \%$ \\
Não sabiam & $14 \%$ \\
\hline
\end{tabular}

\section{DISCUSSÃO}

É indiscutível a importância do conhecimento farmacológico pelo cirurgião-dentista, tendo em vista que é comum a prescrição de medicamentos em suas consultas. A farmacologia constitui-se hoje um ramo bastante relevante no meio odontológico. $\mathrm{O}$ adequado conhecimento da farmacologia pelos profissionais é de grande valia para um tratamento completo e eficiente, sem danos aos pacientes.

A preocupação com que as universidades transmitem os conhecimentos sobre as terapias medicamentosas já foi fonte de estudos como o de Costa et al. ${ }^{2}$ que buscou avaliar os estudantes do último ano de uma Faculdade de Odontologia privada no estado da Paraíba. A autora expõe a necessidade de se reavaliarem os conteúdos de Farmacologia no currículo do curso de graduação em Odontologia, pois $45 \%$ dos entrevistados disseram se sentir inseguros e terem dúvidas frequentes com relação à prescrição medicamentosa. Para autora, as universidades não obtém êxito na transmissão dos conhecimentos sobre os medicamentos, pois se observa uma formação muito aquém do necessário para boa prescrição. Esses dados corroboram os resultados encontrados nesse estudo, já que a maioria dos profissionais (62\%) respondeu que os ensinamentos de Farmacologia/Terapêutica foram insuficientes para um bom exercício profissional.

A utilização dos antimicrobianos em Odontologia deve ser sempre como tratamento auxiliar das infecções orais ou maxilofaciais, pois na sua grande maioria o tratamento definitivo é a remoção do agente causal, o que finaliza o tratamento, evitando superdosagens desnecessárias, além de prevenir a resistência bacteriana aos fármacos mais comumente utilizados por uso incorreto por parte dos pacientes ou indicação desnecessária dos profissionais ${ }^{6,7}$.

No que se refere à escolha dos profissionais atuantes no munícipio de Patos - PB quanto aos antimicrobianos, uma parcela bastante significativa opta pela amoxicilina, correspondendo a $93 \%$ dos entrevistados, o que está de acordo com a pesquisa feita por Santos et al. ${ }^{7}$, entrevistando cirurgiões-dentistas da cidade de Belo Horizonte - MG, na qual a amoxicilina é citada como o antimicrobiano mais prescrito por $93 \%$ dos entrevistados. De acordo ainda com o estudo de Abrantes et al. $^{1}$, a amoxilicina também é o antimicrobiano mais receitado entre os receituários emitidos nas unidades básicas de Belo Horizonte. Este autor afirma que a prescrição frequente pode ser justificada pelo fato de as penicilinas possuírem baixa toxicidade, poucos efeitos colaterais e baixo custo, sendo muito eficiente em vários tipos de processos infecciosos.

De acordo com o presente estudo, a preferência dos profissionais entrevistados com relação aos anti-inflamatórios mais prescritos foi a nimesulida $(55 \%)$. Barreto et al. ${ }^{2}$ também afirmam em seu estudo que os anti-inflamatórios de maior prescrição no consultório odontológico são os inibidores da COX-2 (ciclooxigenase), da qual faz parte a nimesulida, por mecanismo de ação mais satisfatório e menores chances de danos gastrointestinais indesejáveis, tendo em vista que os medicamentos inibidores da COX-1 inibem a secreção do muco protetor gástrico e aumentam o risco de sangramento cutâneo e gastrointestinal. Já a COX-2, apesar de estar presente em diversos órgãos, não se associa a plaquetas, o que torna menor o risco de sangramentos e danos. Porém, a COX-2 também apresenta efeitos adversos no sistema cardiorrespiratório, mas em menor grau ${ }^{9}$.

O tratamento odontológico durante a gravidez ainda é algo mistificado para a população. Ter conhecimento sobre quais materiais e fármacos utilizar ajudam a prevenir futuras iatrogenias que podem trazer danos não apenas a gestante, mas também ao feto, tornando assim os procedimentos mais seguros. Dado preocupante foi encontrando no presente estudo, onde $8 \%$ dos cirurgiões-dentistas responderam que o sal anestésico não indicado para uso em pacientes gestantes é a lidocaína, sendo esse o anestésico apontado na literatura como o mais seguro para realizar procedimentos em pacientes em tais condições ${ }^{10}$.

De forma geral, quando perguntados sobre a importância da Farmacologia para sua vida profissional, 97\% afirmaram entender que a farmacologia constitui-se de grande valia para o exercício profissional, sendo de extrema importância que os profissionais de saúde se revistam de conhecimento nesta área para que possam desempenhar suas atividades de um modo mais seguro e eficaz ${ }^{10,11}$. Seja em procedimentos básicos em consultório odontológico ou em unidades hospitalares, a correta indicação e administração dos fármacos em Odontologia é imprescindível como meio de tratar e manter o tratamento dos pacientes atendidos, para que o funcionamento da clínica odontológica ocorra de maneira mais segura e resolutiva ${ }^{11}$.

\section{CONCLUSÃO}

O nível de conhecimento científico evidenciado pelos Cirurgiões-Dentistas do município de Patos-PB necessita de aprimoramento, notando-se que alguns profissionais encontram-se despreparados na área de farmacologia e terapêutica, sendo alguns incapazes de realizar prescrições adequadas, o que configura um risco para a saúde dos pacientes. 


\section{REFERÊNCIAS}

1. Costa MM. Análise das prescrições de antimicrobianos: farmácia pública da prefeitura de Carmo do Cajuru, Minas Gerais, Brasil. Rev Saúde e Desenvolvimento. 2016; 9(5):73-84.

2. Gaujac C, Oliveira AN, Barreto FAM, Salgado LM, Oliveira MS, Girão RS. Reações alérgicas medicamentosas no consultório odontológico. Rev Odontol Univ São Paulo. 2009; 21(3):268-76.

3. Borges KCAV, Silva PCO, Peixoto FB, Nogueira RVB, Peixoto MOB. Terapêutica medicamentosa em odontologia para pacientes portadores de asma. RvAcBO. 2018; 27(1):17-24.

4. Santos LCA, Faria JMP, Andrade JDS, Souza LG, Fernandes MAB, Botelho PM et al. Avaliação da antibiticoterapia na Odontologia. Arq Bras Odontol. 2015; 11(2):1-6.

5. Costa SANL, Castro RD, Oliveira JA, Cardoso ANS. Prescrição medicamentosa: análise sobre o conhecimento dos futuros cirurgiões-dentistas. Rev bras odontol. 2013; 70(2):172-7.

6. Abrantes PM, Magalhães SMS, Acúrcio FS, Sakurai E. Avaliação da qualidade das prescrições de antimicrobianos dispensados em unidades públicas de saúde de Belo Horizonte, Minas Gerais, Brasil, 2002. Cad Saúde Pública. 2007; 23(1):95-104.

7. Silva RNF, Pereira LCG. O uso de antiinflamatórios esteroidais e não esteroidais no controle da dor e do edema em cirurgia de terceiros molares. Rev Bahiana Odonto. 2016;1(7):31-9.

8. Barreto RC, Diniz MFFM, Pereira GAS, Celani HRB. Antiinflamatórios não hormonais na clínica médicaodontológica. R bras ci Saúde. 2017; 21(1):97-102.

9. Souza FCB, Silva MZM. Controle do processo inflamatório na odontologia com anti-inflamatorios nãoesteroidais. Uningá Review. 2014; 20(2):35-42.

10. Bastos RDS, Silva BS Cardoso JA, Farias JG, Falcão GGVCS. Desmistificando o atendimento odontológico a gestante: Revisão de literatura. Rev Bahiana Odonto. 2014; 5(2):104-6.

11. Maia JA, Silva Alves TM, Boer NP, Correia TM, Motta AL, Cunha-Correia AS. Sedação mínima com midazolam em Odontopediatria: relato de caso de retratamento endodôntico. Arch Health Invest. 2018; 7(1):4-11.

\section{CONFLITO DE INTERESSES}

Os autores declaram não haver conflitos de interesse.

\section{AUTOR PARA CORRESPONDÊNCIA}

Moan Jéfter Fernandes Costa

moanjefter@gmail.com

Submetido em 01/03/2018

Aceito em 09/04/2018 